\title{
Hydroxysafflor yellow A attenuates oxidative stress injury-induced apoptosis in the nucleus pulposus cell line and regulates extracellular matrix balance via CA XII
}

\author{
SHUO YANG and WENBO LIAO \\ Department of Orthopedic Surgery, Affiliated Hospital of Zunyi Medical University, Zunyi, Guizhou 563003, P.R. China
}

Received October 23, 2021; Accepted November 30, 2021

DOI: $10.3892 /$ etm.2021.11105

\begin{abstract}
Intervertebral disc degeneration (IVDD) is the main cause of lower back pain. Oxidative stress injury and degradation of the extracellular matrix (ECM) are important factors causing IVDD, while hydroxysafflor yellow A (HSYA) has significant anti-oxidative stress and anti-apoptotic effects. The present study aimed to investigate the protective role of HSYA in IVDD using nucleus pulposus (NP) cells. A Cell Counting Kit-8 assay was used to detect cell viability following HSYA and tert-Butyl hydroperoxide (TBHP) treatment. Cellular reactive oxygen species levels and the level of apoptosis were measured using flow cytometry. The concentration of superoxide dismutase (SOD), malondialdehyde (MDA), catalase (CAT) and glutathione peroxidase GSH-Px were detected using ELISA. DAPI staining was performed for nuclear morphology analysis, while western blot analysis was used to detect apoptotic- and ECM-related protein expression levels. Bioinformatics analysis was used to predict the binding site between HSYA and carbonic anhydrase 12 (CA12; CA XII). NP cells were transfected withsmall interference RNA (siRNA) for CA XII downregulation. Following TBHP treatment, the level of ROS increased significantly, and the concentrations of SOD, CAT and GSH-Px were decreased. In addition, the apoptosis level of the NP cell line significantly increased following TBHP treatment. Furthermore, the expression levels of ECM-related proteins, collagen II and aggrecan were significantly decreased, and the protein expression level of MMP-13 was significantly increased. HSYA $(10 \mu \mathrm{M})$ could effectively alleviate the effects of TBHP on NP cell apoptosis, oxidative stress damage and the expression level of ECM-related proteins. A binding site was found between HSYA and CA XII. In addition, CA XII-siRNA significantly reduced the increase in the expression level of collagen II
\end{abstract}

Correspondence to: Dr Wenbo Liao, Department of Orthopedic Surgery, Affiliated Hospital of Zunyi Medical University, 149 Dalian Road, Zunyi, Guizhou 563003, P.R. China

E-mail: liaowb1127@163.com

Key words: intervertebral disc degeneration, oxidative stress injury, hydroxysafflor yellow A, carbonic anhydrase 12 and aggrecan proteins and decrease in the expression level of MMP-13 induced by HSYA in the NP cell line. In conclusion, HSYA could attenuate oxidative stress injury and apoptosis induced by TBHP in the NP cell line, and could improve the regulation of ECM balance.

\section{Introduction}

Intervertebral disc degeneration (IVDD) is a degenerative disease and is the leading cause of lower back pain and disability worldwide (1,2). IVDD is also the most common cause of injury and disability in older adults, with symptoms such as stiff spine, stiff neck and back pain (2). The aging population exacerbates the incidence rate and economic burden of IVDD (3). It is well-known that nucleus pulposus (NP) dysfunction has been associated with IVDD. In NP tissues, NP cells play an essential role in maintaining extracellular matrix (ECM) homeostasis (4-6). It has been demonstrated that excessive apoptosis of the NP cells, caused by oxidative stress, inflammation and mechanical load, which subsequently leads to exacerbated ECM degradation, could be a critical pathogenic mechanism of disc degeneration (7). Oxidative stress typically refers to tissue damage caused by the accumulation of reactive oxygen species (ROS) in cells and tissues following the production of free radicals in the body and exceeds its scavenging rate (8). Therefore, the balance is broken. ROS plays a vital role in the development of many degenerative diseases, including intervertebral disc degeneration, neuropathy (9), diabetes (10), senile dementia (11) and degenerative eye disease (12). It has been shown that high levels of ROS in the intervertebral disc not only led to NP cell apoptosis, but also accelerated cell senescence, ultimately leading to disc degeneration (13). Therefore, reducing oxidative stress in NP cells could slow ECM degradation and may be key to improving disc degeneration.

Carthamus tinctorius $L$, also known as grass safflower, is a dried flower of Compositae plants. It is a type of traditional Chinese medicine that promotes blood circulation and provides pain relief. Hydroxysafflor yellow A (HSYA), a mono chalcone glycoside derived from safflower, is the main component of safflower yellow pigment (14). Lee et al (15) found that HSYA could protect the kidney by inhibiting oxidative stress injury and renal cell apoptosis induced by nephropathy in type 2 diabetic rats. In addition, HSYA could protect neuronal cells 
from cellular damage induced by oxidative stress in rats with traumatic brain injury (14). Furthermore, HSYA also plays a role in the regulation of ECM balance. HSYA could reduce the protein expression level of matrix metalloproteinase 13 (MMP-13) and thrombospondin motif 5 (ADAMTS5) to attenuate IL-1 $\beta$-induced ECM degradation, thereby delaying the lipopolysaccharide-induced increase in disc matrix metabolism and inflammatory response, and playing a protective role (16). Therefore, we hypothesized that HSYA may alleviate the degenerative process by improving anti-oxidative stress and the ECM balance regulation ability of the NP cells.

Tert-butyl hydroperoxide (TBHP) is an exogenous inducer of oxidative stress. Due to its high stability and sustained release ability, it has been used in various models of degenerative diseases, including NP cell osteoarthritis oxidative stress models (17). HSYA is considered to exert good antioxidant activity and to regulate ECM balance. In the present study, cell experiments were conducted to verify whether HSYA could inhibit the oxidative stress-induced apoptosis of nucleus pulposus cells and collagen degradation by activating CA XII, thus weakening IVDD. CA XII plays a vital role in intervertebral disc degeneration (18-20).

\section{Materials and methods}

Primary cell culture of the NP cells. NP tissue was obtained from 4-week-old Sprague-Dawley rats (sacrificed by cervical dislocation). NP tissues were digested with $0.2 \%$ collagenase type II (Beijing Solarbio Science and Biotechnology Co., Ltd.) for $25 \mathrm{~min}$ at $37^{\circ} \mathrm{C}$, filtered through a 100 -mesh cell filtration screen, resuspended in DMEM/F12 [Gibco; Thermo Fisher Scientific, Inc.; containing 10\% FBS (Hyclone; Cytiva) and 1\% penicillin/streptomycin], and placed in an incubator at $37^{\circ} \mathrm{C}$ with $5 \% \mathrm{CO}_{2}$. Subcultures were performed when the NP cells reached $80-90 \%$ confluence. All protocols conformed to the Guide for the Care and Use of Laboratory Animals published by the American Physiological Society (21). The current study was approved by the Affiliated Hospital of Zunyi Medical University Ethics Committee for the Use of Experimental Animals (approval no. KLL-2020-317).

NP cell identification. The primary NP cells were cultured in 6 -well plates, at a density of $1 \times 10^{5}$, and a coverslip was placed in the bottom layer for cell climbing. After $24 \mathrm{~h}$, the coverslips were removed and the NP cells were washed with cold PBS, fixed in $4 \%$ paraformaldehyde at $4^{\circ} \mathrm{C}$ for $15 \mathrm{~min}$, permeabilized with $0.5 \%$ Triton X-100 for $20 \mathrm{~min}$, then blocked with $1 \%$ BSA (Proliant Biologicals, LLC) for $30 \mathrm{~min}$ at room temperature. Next, the samples were incubated with primary antibodies against collagen II (1:100; cat no. ER1906-48) and aggrecan (1:50; cat no. ET1704-57) (both from Huabio) overnight at $4^{\circ} \mathrm{C}$. The following day, the samples were incubated with HRP-labeled goat anti-rabbit IgG antibody (1:100; cat no. HA1014; Huabio) for $1 \mathrm{~h}$ at room temperature, then stained with DAPI at room temperature for $10 \mathrm{~min}$. Finally, images were captured under a fluorescence microscope (DM500; Leica Microsystems, Inc.).

Cytotoxicity assay. According to the manufacturer's instructions, cell viability was measured using a Cell Counting
Kit (CCK)-8 assay (cat no. C0037; Beyotime Institute of Biotechnology). The NP cell line was seeded in 96-well plates, at a density of $5 \times 10^{3}$ cells/well, and incubated with different concentrations $(0,5,10,15,20,25,30,35,40,45$ and $50 \mu \mathrm{M})$ of HSYA (cat no. 78281-02-4) and TBHP (0, 20, 40, 60, 80, $100,120,140,160,180$ and $200 \mu \mathrm{M}$ ), cat no. 75-91-2) (both from Shanghai Macklin Biochemical Co., Ltd.) for $24 \mathrm{~h}$. CCK-8 solution $(10 \mu \mathrm{l})$ was added to each well and the NP cells were cultured for another $4 \mathrm{~h}$ at $37^{\circ} \mathrm{C}$. The absorbance of each sample was measured at $450 \mathrm{~nm}$ using a microplate reader (Thermo Fisher Scientific, Inc.).

Cellular model of oxidative stress. The primary NP cell line was subjected to oxidative stress model induction using TBHP $(40 \mu \mathrm{M})$. The cells treated with TBHP only were used as the model group. The cells treated with HSYA $(10 \mu \mathrm{M})$ and TBHP $(40 \mu \mathrm{M})$ were the HSYA group. The control group was untreated.

ROS detection using flow cytometry. A ROS assay kit (cat no. CA1410; Beijing Solarbio Science and Technology, Co., Ltd.) was used to detect intracellular ROS levels. NP cells (treated with $40 \mu \mathrm{M}$ THBP or $10 \mu \mathrm{M}$ HSYA) were incubated with 7-dichloro-dihydrofluorescein diacetate at a final concentration of $10 \mu \mathrm{M}$ at $37^{\circ} \mathrm{C}$ for $30 \mathrm{~min}$, and were mixed upside down every $5 \mathrm{~min}$. The NP cells were then washed three times with cold PBS and analyzed using flow cytometry (Attune NxT; Applied Biosystems; Thermo Fisher Scientific, Inc.). Flow cytometry results were analyzed using FlowJo $^{\text {TM }}$ v10.8 Software (BD Biosciences).

ELISA. NP cells (treated with $40 \mu \mathrm{M}$ THBP or $10 \mu \mathrm{M}$ HSYA) were collected, then cell lysis was performed using cell lysis buffer (Zhejiang Ruyao Biotech Co., Ltd.). Following centrifugation at $2,000 \mathrm{x} \mathrm{g}$ at room temperature for $5 \mathrm{~min}$, the pellet was removed and the supernatant was collected for further use. The superoxide dismutase (SOD; cat. no. BC0170), malondialdehyde (MDA; cat. no. BC0025), catalase (CAT; cat. no. BC0200), and glutathione peroxidase (GSH-Px; cat. no. BC1175) concentrations were detected according to the manufacturer's instructions (Beijing Solarbio Science and Technology, Co., Ltd.).

Analysis of apoptosis. An Annexin V-FITC/PI apoptosis kit (cat no. 70-AP101-100; Multi sciences (Lianke) Biotech Co., Ltd.) was used in this experiment. The NP cells $\left(5 \times 10^{5}\right)$ were seeded in 6-well plates for cell culture overnight at $37^{\circ} \mathrm{C}$. The cells were treated according to the aforementioned groups. After incubation with $40 \mu \mathrm{M}$ TBHP and $10 \mu \mathrm{M}$ HSYA for $24 \mathrm{~h}$, the cell supernatant was removed to preserve the floating cells. Adherent cells were digested with trypsin and washed with PBS. The cell precipitates were obtained after centrifugation at $800 \mathrm{x} \mathrm{g}$ at room temperature for $5 \mathrm{~min}$ and then suspended in PBS. They were washed twice with precooled PBS and resuspended in binding buffer, at a final concentration of $1 \times 10^{6}$ cells $/ \mathrm{ml}$. A total of $5 \mu \mathrm{l}$ Annexin V-FITC and $5 \mu \mathrm{l}$ PI were added to $100 \mu 1$ cells containing $1 \times 10^{5}$ cells. The cells were mixed by gentle rotation and incubated in the dark at room temperature for $15 \mathrm{~min}$. A total of $400 \mu \mathrm{l}$ binding buffer was added for analysis using a Life Attune sonic focusing flow 
cytometer (Attune NxT; Applied Biosystems; Thermo Fisher Scientific, Inc.). The flow cytometry results were analyzed using FlowJo ${ }^{\text {TM }}$ v10.8 Software (BD Biosceinces). All the samples were performed in triplicate and repeated three times.

Nuclear morphological analysis. The NP cells $\left(3.5-5 \times 10^{5}\right)$ were seeded in 6-well plates for cell culture at $37^{\circ} \mathrm{C}$ and a coverslip was placed at the bottom of the plate for cell climbing, and attachment overnight. The cells were treated according to the aforementioned groups. After $24 \mathrm{~h}$, the coverslips were removed, fixed with methanol precooled at $4^{\circ} \mathrm{C}$ for $10 \mathrm{~min}$ and permeabilized for $10 \mathrm{~min}$. After washing three times with PBS containing $0.5 \%$ Tween-20, they were washed once with PBS, followed by staining with $1 \mu \mathrm{g} / \mathrm{ml}$ DAPI (dissolved in PBS) reagent at room temperature for $10 \mathrm{~min}$. Subsequently, the samples were washed three times with PBS, followed by an anti-fluorescence quencher and mounted with neutral gum. The results were analyzed using a fluorescence microscope (DM500; Lecia Microsystems, Inc.). The number of nuclear pyknotic cells in three different visual fields was counted by fluorescence microscopic observation (magnification, $\mathrm{x} 400$ ).

Western blot analysis. The treated NP cells were lysed on ice using RIPA buffer (cat no. P0013B; Beyotime Institute of Biotechnology), containing protease and phosphatase inhibitors (100:1 by volume). The supernatant was obtained following centrifugation at $10,000 \times \mathrm{g}$ for $10 \mathrm{~min}$ at $4{ }^{\circ} \mathrm{C}$, then the samples were quantified using a BCA protein assay kit (Beyotime Institute of Biotechnology). Total protein $(20 \mu \mathrm{g})$ from each treatment group were separated using $10 \%$ SDS-PAGE and transferred to PVDF membranes. After blocking with $5 \%$ skimmed milk for $2 \mathrm{~h}$ at room temperature, the membranes were incubated with primary antibodies against Bcl-2 (1:2,000; cat. no. 60178-1-Ig; ProteinTech Group, Inc.), Bax (1:2,000; cat. no. 60267-1-Ig; ProteinTech Group, Inc.), caspase-3 (1:1,000; cat. no. 9662S; Cell Signaling Technology, Inc.), cleaved caspase-3 (1:1,000; cat. no. 9664T; Cell Signaling Technology, Inc.), caspase-9 (1:1,000; cat. no. A2636; ABclonal Biotech Co., Ltd.), cleaved caspase-9 (1:1,000; cat. no. ER60008; Huabio) MMP-13 (1:1,000; cat. no. 18165-1-AP; ABclonal Biotech Co., Ltd.), collagen II (1:1,000; cat. no. ER1906-48; Huabio), aggrecan (1:1,000; cat. no. ET1704-57; Huabio), CA XII (1:1,000; cat. no. ER62856; Huabio), and $\beta$-tubulin $(1: 1,000$; cat. no. 2128S; Cell Signaling Technology, Inc.) overnight at $4^{\circ} \mathrm{C}$. Subsequently, the membranes were incubated with goat anti-rabbit IgG/HRP antibody (1:2,000; cat. no. SE134; Beijing Solarbio Science and Technology, Co., Ltd.) at room temperature for 2 h. An ECL kit (cat. no. SW2030; Beijing Solarbio Science and Technology, Co., Ltd.) was used analyze the expression level along with a UVP gel imager. ImageJ v1.8.0 software (National Institutes of Health) was used for densitometry.

HSYA target protein prediction. The 3D structure of HSYA was downloaded from the Chemspider database (http://www. chemspider.com/). The 3D structure of the protein was obtained from the RCSB Protein Data Bank (PDB; http://www.rcsb.org/). The target proteins predicted by HSYA were intersected with genes associated with disc degeneration to determine the most likely target proteins. The remaining target proteins were simulated and constructed using Pymol software (v2.3.0 software (Schrodinger Technologies Inc.). Pymol software was used to remove water molecules and obtain ligands from the protein structure, which were stored in PDBQT format, respectively. Molecular docking analysis was performed using AutoDock 4 (https://autodock.scripps.edu/). Depending on the location of the protein ligand, the protein active site coordinates were determined and default values were used for all other parameters. Eventually, the conformation with the lowest docking binding energy was selected for docking binding mode analysis and plotted using the visualization function of the Pymol molecular simulation software.

Reverse transcription-quantitative PCR (RT-qPCR). Total RNA, from the treated cells, was extracted using TRIzol ${ }^{\circledR}$, then the Reverse transcription kit (cat. no. E047-01B; Novoprotein Technology Co., Ltd.) was used to generate cDNA. After adding the components according to the instructions, samples were incubated at $25^{\circ} \mathrm{C}$ for $5 \mathrm{~min}$. Then it was incubated at $42^{\circ} \mathrm{C}$ for $30 \mathrm{~min}$. Subsequently, CA XII expression level was detected using a NovoStart ${ }^{\circledR}$ SYBR qPCR SuperMix Plus kit (Novoprotein Technology Co., Ltd.) according to the manufacturer's instructions. The relative expression level of CA XII was calculated using the $2^{-\Delta \Delta \mathrm{Cq}}$ method (22) and $\beta$ III-tubulin as an internal reference. Each sample was analyzed in triplicate. The following primers were used: CA XII forward, 5'-TGG ACCTACATTGGTCCTGC-3', and reverse, 5'-GCT CTGTAC TGGTGAGGCTG-3'; and $\beta$ III-tubulin forward, 5'-CAATGA GGCCTCCTCTCACA-3, $\beta$ III-tubulin and reverse, 5'-TGT ATAGTGCCCTTTGGCCC-3'.

CA XII knockdown using small interfering RNA (siRNA). The coding sequence of the CA XII mRNA was downloaded from National Center for Biotechnology Information (https://www. ncbi.nlm.nih.gov/), and the CA XII siRNA sequences were designed using siDirect version 2.0 (http://sidirect2.rnai.jp/), and the three top-scoring sequences were used for validation. The primers were synthesized by Jiangsu Genewiz Biotechnology Co., Ltd. The cells were plated in 24-well plates to ensure that transfection was performed when the confluence of the cells was $30 \%$. The siRNA duplexes (6 pmol) were first solubilized and diluted with $50 \mu \mathrm{l}$ serum-free Opti-MEM, then $1 \mu$ l Lipofectamine ${ }^{\circledR}$ RNAimax mixture (cat no. 13778030; Thermo Fisher Scientific, Inc.) was added for $10 \mathrm{~min}$ at room temperature. The siRNA duplex/RNAimax complex was added to the cell transfection medium at a final siRNA concentration of $10 \mathrm{nM}$ and co-incubated at $37^{\circ} \mathrm{C}$ for $48 \mathrm{~h}$. After $48 \mathrm{~h}$, the gene and protein expression levels of CA XII were examined using RT-qPCR and western blot analysis to verify successful knockdown of expression. The following siRNA sequences were used: siRNA1 forward, 5'-UUCAAU AGC UUUUCAACAGAC-3' and reverse, 5'-CUGUUGAAA AGCUAUUGAACC-3'; siRNA2 forward, 5'-AUUGGUUAG GUUCAAUAGCUU-3' and reverse, 5'-GCUAUUGAACCU AACCAAUGA-3'; siRNA3 forward, 5'-ACUGAAUGGCCA UCAUUGGUU-3' and reverse, 5'-CCAAUGAUGGCCAUU CAGUGA-3'; and negative control siRNA (non-targeting sequence) forward, 5'-AUCGUACGUACCGUCGUAU-3' and reverse, 5'-AUACGACGGUACGUACGAU-3'. 
A
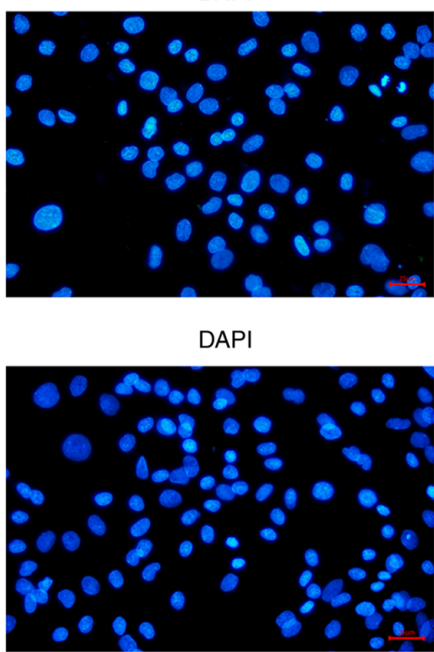

B

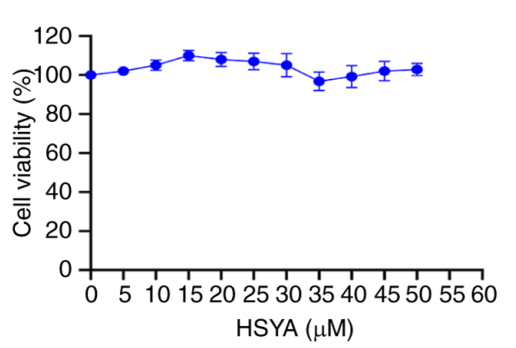

Collagen II

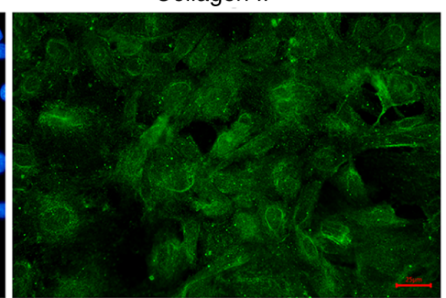

Aggrecan

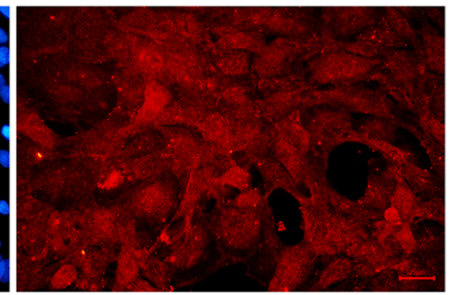

c

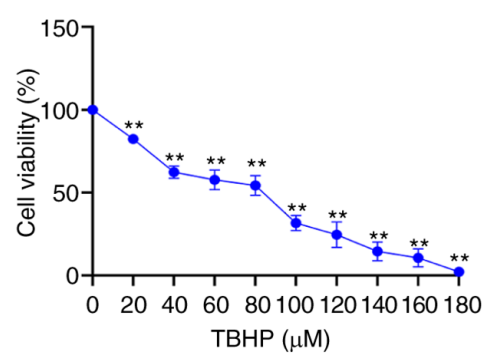

Merged

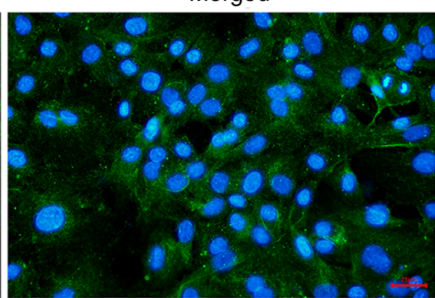

Merged
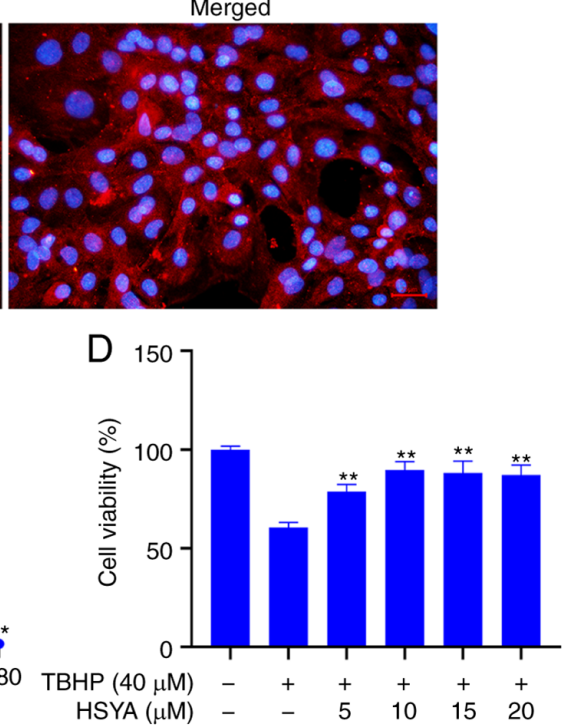

Figure 1. Determination of drug concentration. (A) Immunofluorescence was used to confirm primary NP cells using collagen II and aggrecan. Scale bar, $25 \mu \mathrm{m}$. A CCK- 8 assay was used to detect the effect of different concentrations of (B) HSYA and (C) TBHP on NP cell viability. ${ }^{* * *}<0.01$ vs. $0 \mu$ M. (D) A CCK- 8 assay was used to detect the effect of TBHP and HSYA on NP cell viability. ${ }^{* *} \mathrm{P}<0.01$ vs. $0 \mu \mathrm{M}$ HSYA+TBHP. HSYA, hydroxysafflor yellow A; NP, nucleus pulposus; TBHP, tert-butyl hydroperoxide; CCK, Cell Counting Kit; DAPI, diamidino-2-phenylindole.

Immunocytochemistry. The primary NP cells were cultured in 6 -well plates, at a density of $1 \times 10^{5}$. A coverslip was placed in the bottom for cell migration, then it was removed after $24 \mathrm{~h}$. The cells were washed with cold PBS, fixed with $4 \%$ paraformaldehyde at $4^{\circ} \mathrm{C}$ for $15 \mathrm{~min}$, permeabilized with $0.5 \%$ Triton X-100 for $20 \mathrm{~min}$. The endogenous peroxidase activity was blocked by incubation with $3 \% \mathrm{H}_{2} \mathrm{O}_{2}$ at room temperature for $20 \mathrm{~min}$, and then blocked with 1\% BSA (Proliant Biologicals, LLC) for $30 \mathrm{~min}$ at room temperature. Next, the samples were incubated with collagen II (1:100, cat. no. ER1906-48; HUABIO), aggrecan (1:50, cat. no. ET1704-57; HUABIO) and MMP-13 (1:50, cat. no. 18165-1-AP; ABclonal Biotech Co., Ltd.) primary antibodies overnight at $4^{\circ} \mathrm{C}$. Subsequently, the samples were incubated with goat anti-rabbit IgG/HRP antibody (1:100, cat. no. SE134; Beijing Solarbio Science \& Technology, Co., Ltd.) for $1 \mathrm{~h}$ at room temperature, stained with diaminobenzidine (DAB, cat. no. P0202; Beyotime Institute of Biotechnology) chromogenic solution (at room temperature for $3 \mathrm{~min}$ ) and hematoxylin (at room temperature for $1 \mathrm{~min}$ ), dehydrated in a gradient alcohol series, immersed in xylene, and mounted with neutral gum. The experimental results were observed and images were captured using a Leica light microscope (magnification, x400; DM500; Lecia Microsystems, Inc.). IPP.6.0 (Image Pro Plus 6.0; Media Cybernetics, Inc.) software was used to quantify the optical density values of the results in each group under three different randomly selected visual fields (magnification, $\mathrm{x} 400$ ) to judge the intensity of positive results.
Statistical analysis. All the experiments were repeated independently three times. The data are expressed as the mean \pm standard deviation. GraphPad Prism 8.0 (GraphPad Software, Inc.) was used to analyze the data. Differences between groups were analyzed using one-way ANOVA followed by Tukey's post hoc test. $\mathrm{P}<0.05$ was considered to indicate a statistically significant difference.

\section{Results}

Effect of HSYA on TBHP-induced oxidative stress injury the NP cells. Disc primary cells were obtained from rat disc NP tissue and cultured. Immunofluorescence validation of the hallmark proteins from the primary NP cells, collagen II and aggrecan, showed that these proteins were highly expressed in the cultured primary cells (Fig. 1A). The cytotoxic effect of HSYA on the NP cells was analyzed using a CCK-8 assay and the results showed that HSYA concentration between 0 , and $50 \mu \mathrm{M}$ did not significantly affect the viability of the cells (Fig. 1B). A CCK-8 assay was also used to detect the effect of different concentrations of TBHP on NP cell viability and the results showed that at $40 \mu \mathrm{M}$ TBHP, cell viability was significantly decreased compared with that in cells treated with $0 \mu \mathrm{M}$ TBHP $(\mathrm{P}<0.01)$ (Fig. 1C). Therefore, $40 \mu \mathrm{M}$ TBHP was selected for subsequent experiments.

Fig. 2A shows the detection of cellular ROS levels, which were analyzed using flow cytometry and further analysis 
A

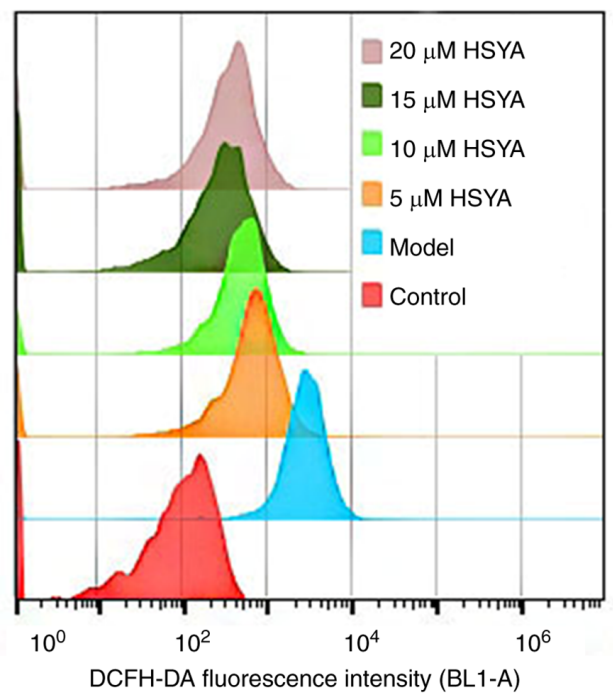

C

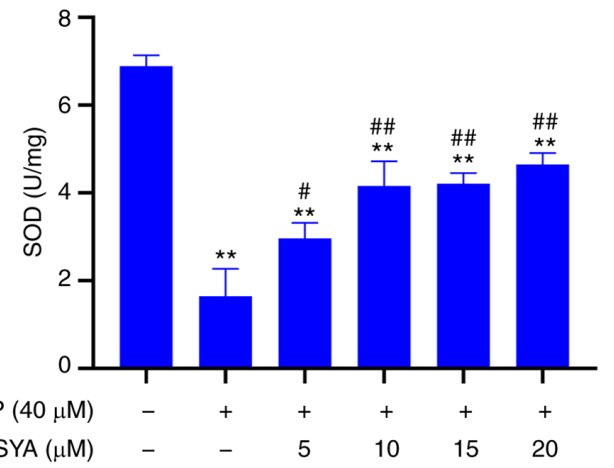

$\mathrm{E}$

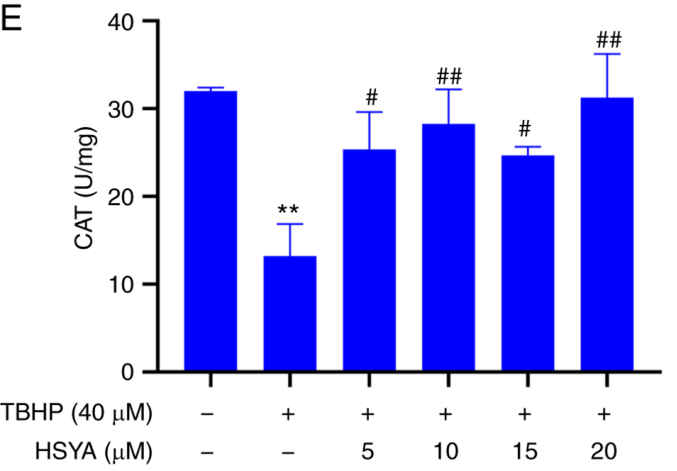

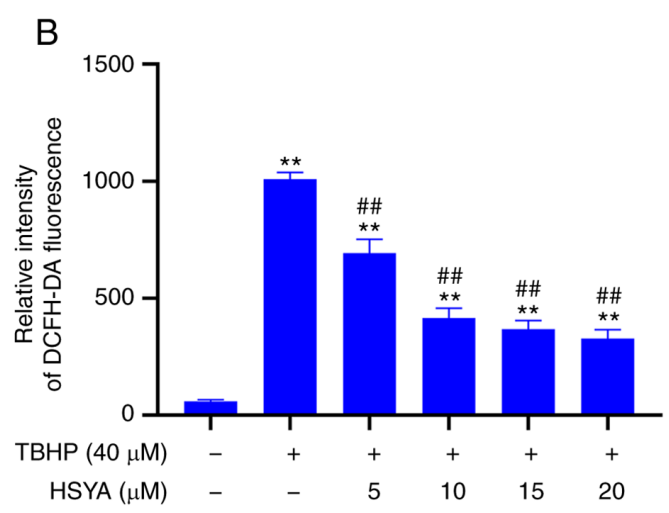
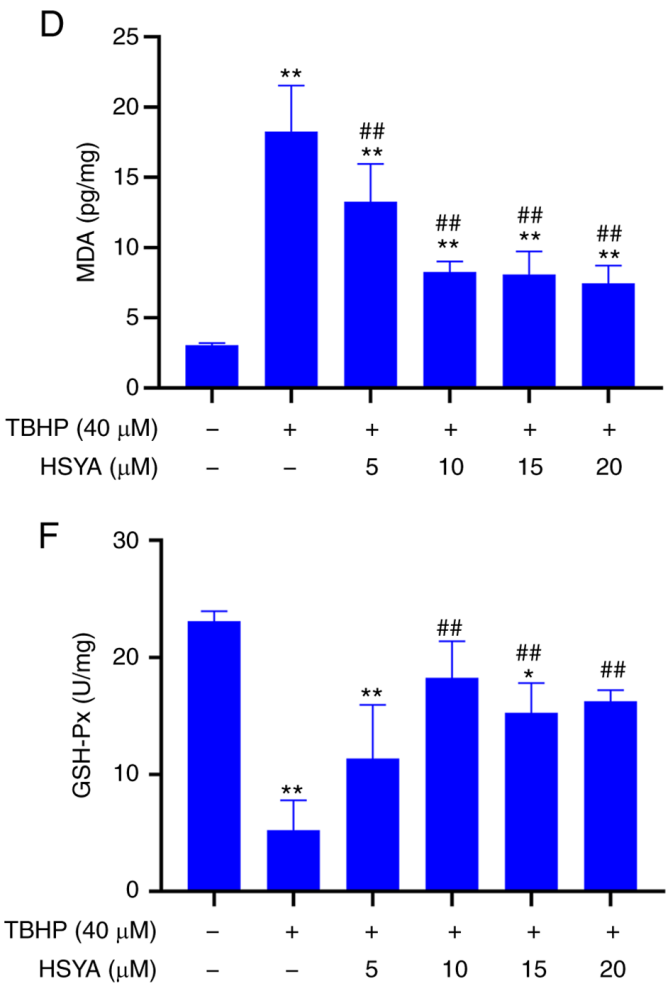

Figure 2. Effect of HSYA on TBHP-induced oxidative stress in NP cells. (A) Reactive oxygen species levels were measured using flow cytometry and the results were (B) statistically analyzed. (C) SOD, (D) MDA, (E) CAT and (F) GSH-Px concentrations were analyzed using ELISA. ${ }^{\text {P }}<0.05$, ${ }^{* *} \mathrm{P}<0.01$ vs. control; ${ }^{\#} \mathrm{P}<0.05,{ }^{\# \#} \mathrm{P}<0.01$ vs. model. HSYA, hydroxysafflor yellow A; NP, nucleus pulposus; TBHP, tert-butyl hydroperoxide; SOD, superoxide dismutase; MDA, malondialdehyde; CAT, catalase; GSH-Px, glutathione peroxidase.

showed that compared with that in the control group, the ROS level in the model group was significantly increased $(\mathrm{P}<0.01)$ (Fig. 2B). However, after administration of 5, 10, 15 and $20 \mu \mathrm{M}$ HSYA, the ROS level was significantly decreased (all $\mathrm{P}<0.01$ ).

The results of oxidative stress index showed that treatment with TBHP could significantly inhibit the concentrations of SOD (Fig. 2C), CAT (Fig. 2E), and GSH-Px (Fig. 2F) in the model group, while the concentrations of MDA (Fig. 2D) were significantly increased (compared with that in the control group, $\mathrm{P}<0.01)$. Compared with that in the model group, the concentrations of SOD, CAT and GSH-Px were significantly increased, and the MDA concentration was significantly decreased following HSYA treatment (all $\mathrm{P}<0.01$ ). With respect to oxidative stress, $10 \mu \mathrm{M}$ HSYA had a greater effect than $5 \mu \mathrm{M}$ HSYA. However, there was no significant difference between 10, 15 and $20 \mu \mathrm{M}$ concentrations of HSYA.

Effect of HSYA on TBHP-induced NP cell apoptosis. The apoptosis level was detected using flow cytometry (Fig. 3A). The statistical analysis showed that TBHP induced apoptosis in the NP cells (Fig. 3B). The apoptosis level in the model group was significantly increased to $15.712 \%$ compared with $4.895 \%$ in the control group $(\mathrm{P}<0.01)$. After treatment with $10 \mu \mathrm{M}$ HSYA, the level of apoptosis decreased to $7.96 \%$, which was significantly lower compared with that in the model group $(\mathrm{P}<0.01)$. Morphology analysis was subsequently performed using DAPI (Fig. 3C). 
A
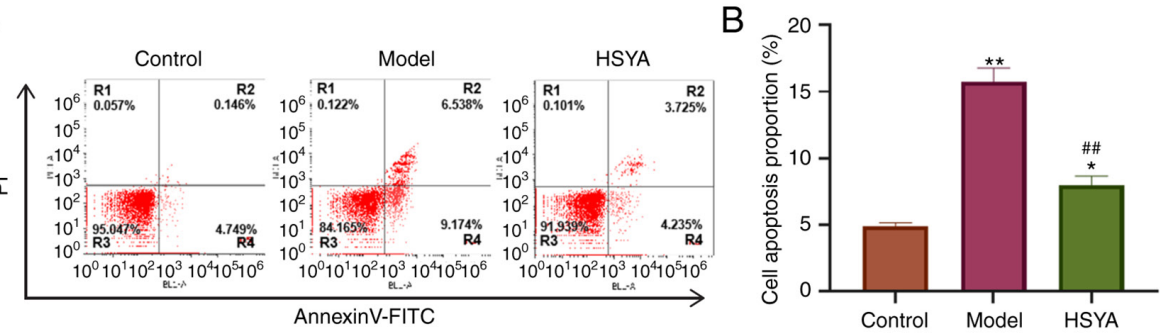

C
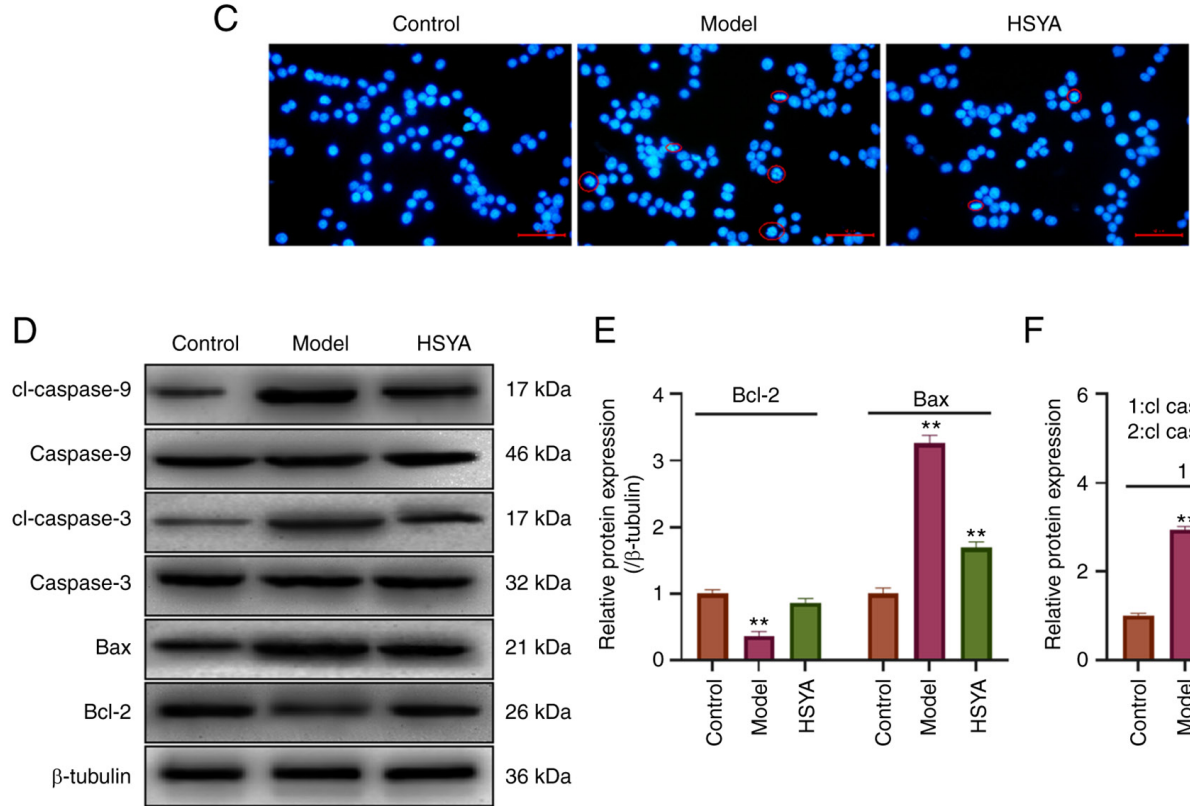

E

$\mathrm{F}$
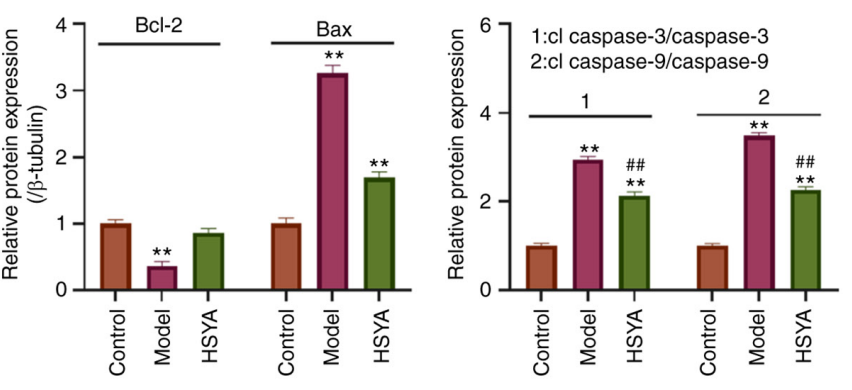

Figure 3. Effect of HSYA on tert-butyl hydroperoxide-induced NP cell apoptosis. (A) Flow cytometry was used to detect the level of apoptosis (B) Diamidino-2-phenylindole was used to stain and analyze nuclear morphology. The red circles indicate pyknotic nuclei and apoptotic bodies. Scale bar, $50 \mu \mathrm{m}$. (C) Statistical analysis of apoptosis detection using flow cytometry. (D) Western blot analysis was used to detect the expression level of apoptosis-related proteins and the results were statistically analyzed for (E) Bcl-2, Bax and (F) cleaved caspase-3 and cleaved caspase-9. ${ }^{*} \mathrm{P}<0.05,{ }^{* *} \mathrm{P}<0.01$ vs. control; ${ }^{\# \#} \mathrm{P}<0.01$ vs. model. HSYA, hydroxysafflor yellow A.

The results showed that the nucleus of the model cell appeared pyknotic, accompanied by the presence of more typical apoptotic bodies (indicated using red circles). The cells showed pyknotic nuclei and there was a decrease in the number of apoptotic bodies following treatment with $10 \mu \mathrm{M}$ HSYA. Apoptosis-related protein expression was detected using western blot analysis (Fig. 3D) and the statistical results showed that compared with that in the control group, the expression of the pro-apoptotic proteins Bax, cleaved-caspase- 3 , and cleaved-caspase- 9 were significantly increased in the model group (all $\mathrm{P}<0.05$ ) (Fig. 3E and F). In addition, the expression level of the anti-apoptotic protein, $\mathrm{Bcl}-2$ was significantly decreased $(\mathrm{P}<0.05)$. Compared with that in the model group, HSYA significantly inhibited the protein expression levels of Bax, cleaved-caspase-3 and cleaved-caspase-9 in the TBHP-induced NP cells, and increased the protein expression levels of $\mathrm{Bcl}-2$ (all $\mathrm{P}<0.05$ ).

Effect of HSYA on ECM-associated proteins. The expression levels of collagen-related proteins were detected using western blot analysis (Fig. 4A). Statistical quantification (Fig. 4B-D) revealed that the expression levels of collagen II and aggrecan protein, which were the markers of NP cells in the model group, were significantly lower than those in control group (both $\mathrm{P}<0.05$ ). In addition, the protein expression levels of MMP-13, a protein related to collagen breakdown (23), were significantly upregulated $(\mathrm{P}<0.05)$. Compared with that in the model group, treatment with HSYA increased the protein expression levels of collagen II and aggrecan in the NP cells, while decreasing the protein expression level of MMP-13, and the difference was statistically significant (all $\mathrm{P}<0.05$ ).

HSYA regulates the expression level of collagen-associated proteins in NP cells via CA XII. Using bioinformatics analysis and Pymol molecular simulation docking software, the interaction between HSYA and CA XII was analyzed (Fig. 5A). The docking results showed that HSYA had a binding site for CA XII. There were six binding bond energies between HSYA and CA XII protein amino acid residues (HIS-117, HIS-91, ALA129 and ASN-64), with a binding energy of $-10.7 \mathrm{kcal} / \mathrm{mol}$, providing a stable spatial structure domain. The protein expression level of CA XII in the treated NP cells was subsequently detected using western blot analysis (Fig. 5B). The densitometry results showed that compared with that in the control group, the protein expression level of CA XII in the model group was significantly inhibited, while the protein expression level of CA XII in the HSYA group was significantly higher compared with that in the model group (all $\mathrm{P}<0.05$ ) (Fig. 5C). The mRNA expression level of the CA XII was also detected using RT-qPCR and the results showed that the mRNA expression level of CA XII in the model group was decreased (compared with that in the control 
A
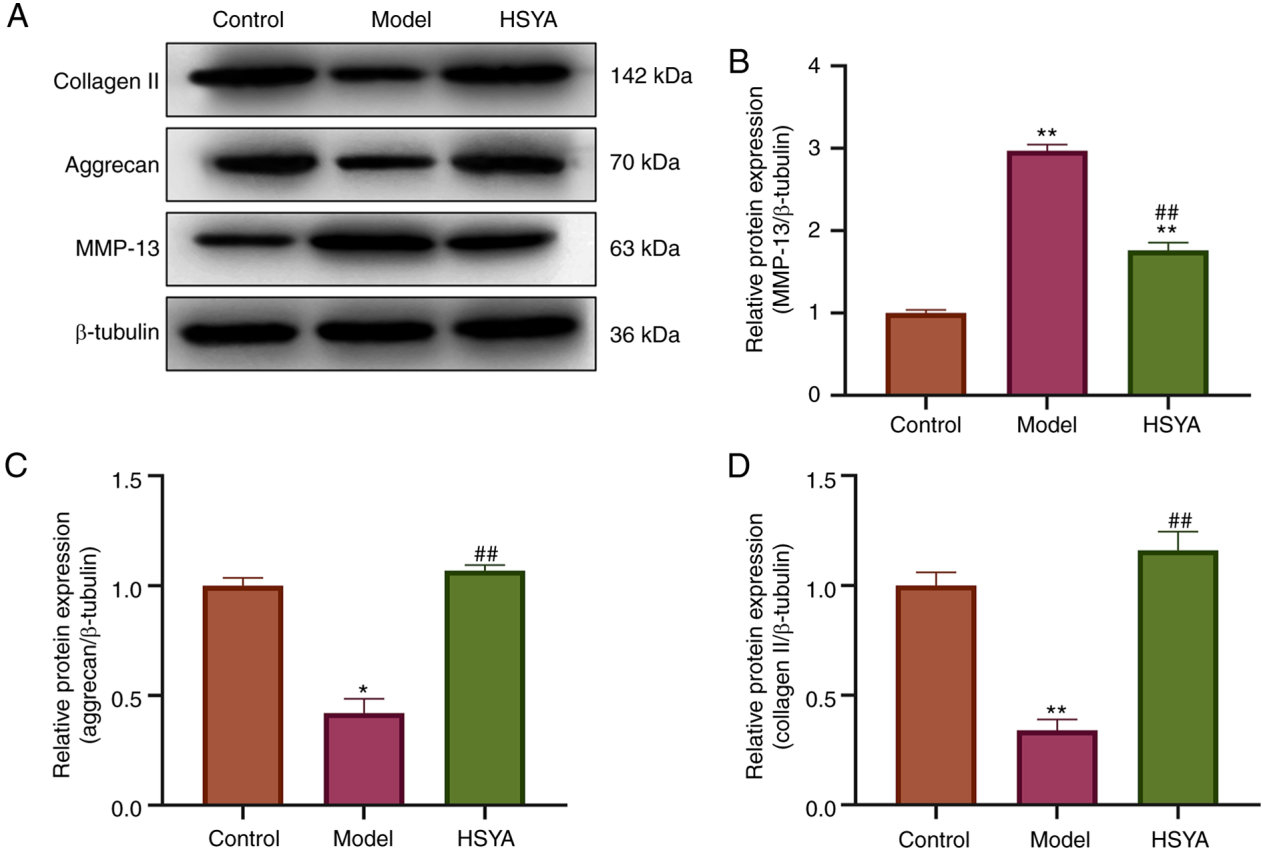

Figure 4. Effect of HSYA on ECM-associated protein expression. (A) Expression levels of ECM-associated proteins were measured using western blot analysis and statistically analyzed for (B) MMP-13, (C) aggrecan and (D) and collagen II. ${ }^{*} \mathrm{P}<0.05,{ }^{* * *} \mathrm{P}<0.01$ vs. control; ${ }^{\# \#} \mathrm{P}<0.01$ vs. model group. ECM, extracellular matrix; HSYA, hydroxysafflor yellow A.

group), while the gene expression level of CA XII in the HSYA group was significantly higher compared with that in the model group (all $\mathrm{P}<0.05$ ) (Fig. 5D). RT-qPCR was also used to investigate the transfection efficiency of siRNA in inhibiting CA XII expression and the results showed that siRNA3 inhibited the expression level more significantly, with a inhibition rate of $77 \%$ (Fig. 5E). These results were confirmed using western blot analysis (Fig. 5F and G). Therefore, siRNA3 was used in subsequent experiments.

The results from western blot analysis, in Fig. 6A and B, showed that si-CA XII could reverse the increase in CA XII protein expression by HSYA. Immunocytochemistry results demonstrated that Aggrecan (Fig. 6C) and collagen II (Fig. 6D) proteins in the HSYA group were higher than those in the Model group, while MMP-13 (Fig. 6E) proteins were lower than those in the TBHP group. However, si-CA12 was able to reverse the effects of HSYA on aggrecan, collagen II and MMP-13 protein expression. Further validation was performed using western blot analysis (Fig. 6F, and the results were consistent with immunocytochemistry. si-CA XII could reverse the increase of aggrecan and collagen II protein expression by HSYA, as well as the inhibitory effect on MMP-13 protein expression (all $\mathrm{P}<0.05$ ) (Fig. 6G-I).

\section{Discussion}

Currently, pharmacological treatment strategies for degenerative disc disease aim to relieve the patients' symptoms. However, it cannot control or reverse the progression of disc degeneration and it produces some side effects $(3,24)$. In the late stages of IVDD, conservative management requires surgical treatment and is accompanied by various surgical risks (25). Excessive apoptosis of disc cells, particularly NP cells, leads to changes in disc structure and function, and is one of the most important pathophysiological mechanisms in the progression of IVDD (7). As a normal byproduct of oxygen metabolism formation, ROS plays a crucial role in homeostasis and cellular signal transduction (26). It has been reported that excess ROS, in the pathogenesis of IVDD, leads to NP cell apoptosis and ECM degradation. Therefore, in the present study, the effect of HSYA on IVDD disease progression was investigated using TBHP oxidative stress injury induction and ROS accumulation. It was found that HSYA could reduce TBHP-induced ROS levels in the NP cells, while increasing the concentrations of the antioxidant enzymes, SOD, CAT and GSH-Px, decrease the expression levels of pro-apoptotic proteins, Bax, cleaved caspase- 3 and cleaved caspase- 9 , and decrease apoptosis in the NP cells. The investigation of HSYA in oxidative stress and anti-apoptosis has been reported in other types of diseases. For example, HSYA reduced oxidative stress and reversed the increase in Bax and caspase- 3 from nephropathy in type 2 diabetic rats, and significantly increased the protein expression of $\mathrm{Bcl}-2$ in renal tissue (15). In addition, in traumatic brain injury in rats, HSYA protected neural cells against oxidative stress and anti-apoptosis by inhibiting the protein expression level of Bax, caspase-3, and caspase-9, while increasing antioxidant enzyme concentrations (14). All of the aforementioned studies confirmed that HSYA has an essential role in anti-oxidative stress and anti-apoptosis.

Furthermore, the degradation of the ECM is also an important factor driving the progression of IVDD (27). The ECM is a non-cellular 3D macromolecular network composed of collagen, proteoglycans/glycosaminoglycans, elastin, fibronectin, laminin and several other glycoproteins (28). While collagen II and aggrecan are important in disc NP cells, their presence provides critical properties for the disc (29). Proteoglycan and type II collagen content in the ECM is reduced when the disc degenerates (30). In addition, when the surrounding biochemical 


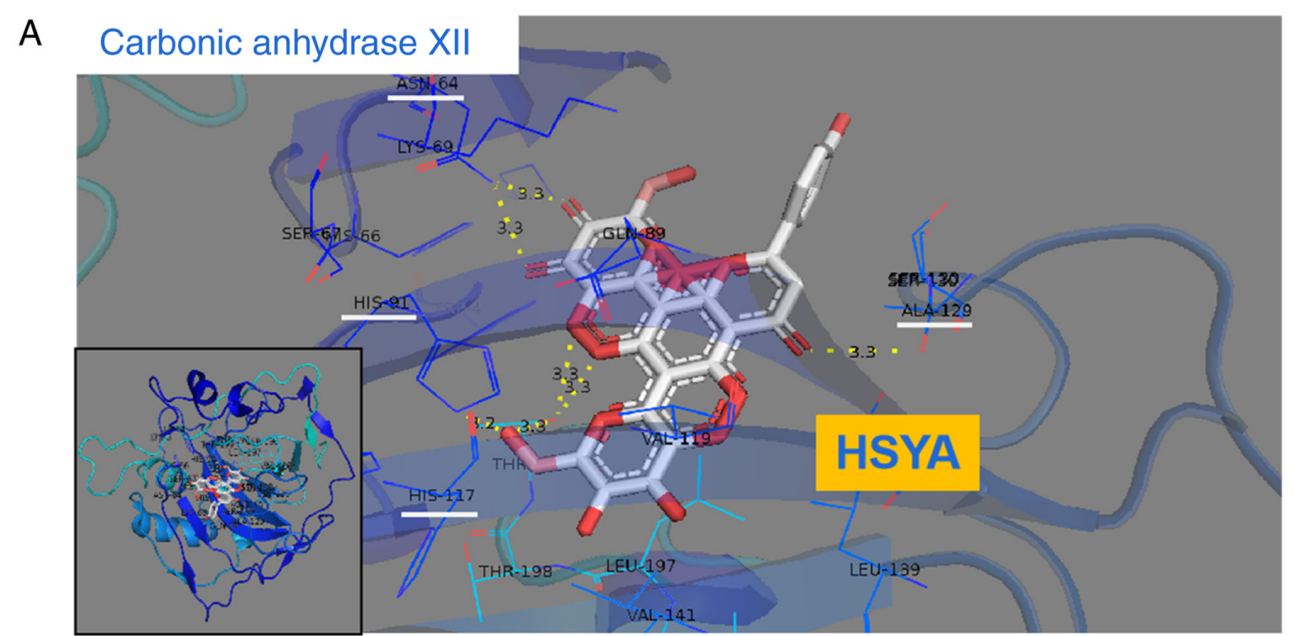

B

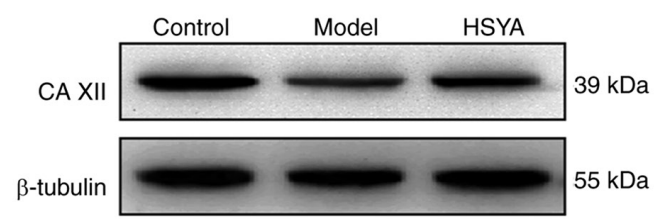

D

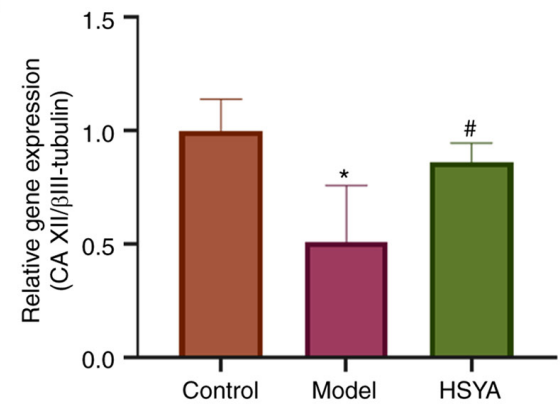

F

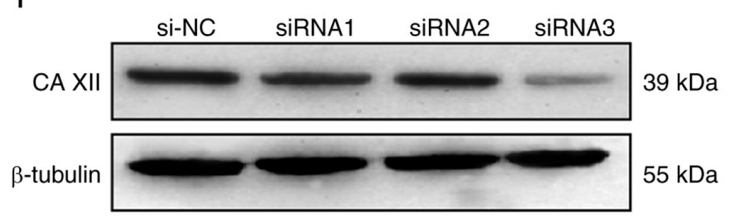

C

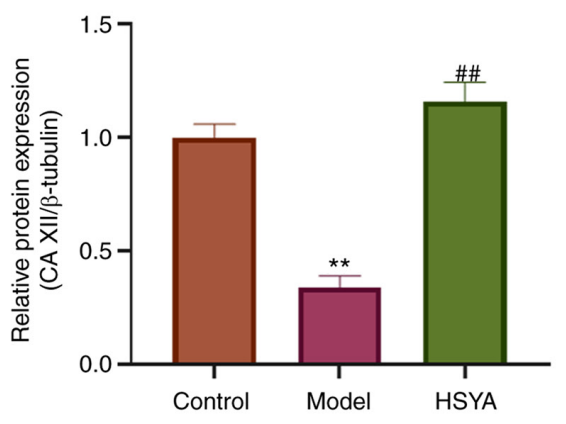

E

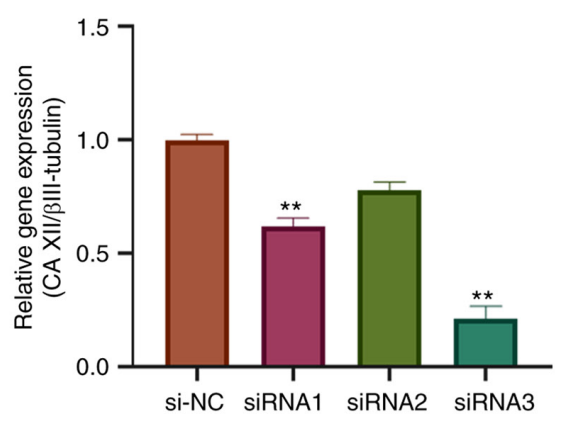

G

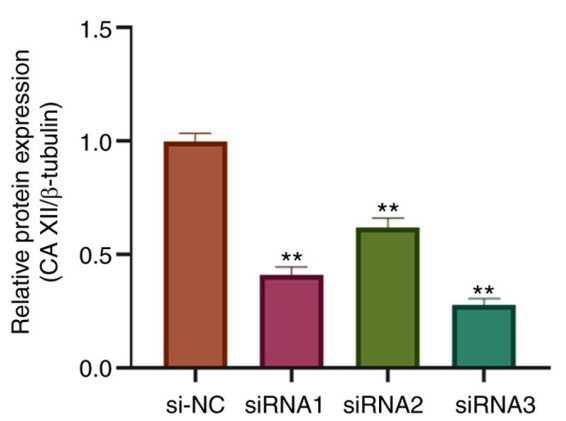

Figure 5. Association between HSYA and CA XII. (A) Visual simulation docking results for the interaction site between HSYA and CA XII protein using Pymol software. (B) Western blot analysis was used to detect the protein expression level of CA XII and the results were (C) statistically analyzed. RT-qPCR was used to detect the mRNA expression level of (D) CA XII and (E) the inhibition rate of CA XII siRNA. (F) Western blot analysis was used to detect the protein expression level of CA XII and the results were $(\mathrm{G})$ statistically analyzed. (C and D) ${ }^{*} \mathrm{P}<0.05,{ }^{* *} \mathrm{P}<0.01$ vs. control; ${ }^{\#} \mathrm{P}<0.05$, ${ }^{\# \#} \mathrm{P}<0.01 \mathrm{vs}$. model; (E and $\mathrm{G}{ }^{* * *} \mathrm{P}<0.01$ vs. si-NC. HSYA, hydroxysafflor yellow A; NC, negative control; si, small interfering; CA XII, carbonic anhydrase 12 .

conditions change, NP cells lose their normal phenotype and turn into mast cells, and replace type II collagen with type X collagen. A previous study confirmed that TBHP could induce ECM metabolic imbalance in the NP cells via the production of large amounts of ROS (31). The in vitro study showed that HSYA significantly increased collagen II and aggrecan protein expression levels in the disc matrix. In addition, ECM metabolism in the NP cells is a dynamic process, including ECM anabolism and ECM catabolism. MMP-13 was shown to be one of the major catabolic factors of ECM in disc degeneration $(32,33)$. 

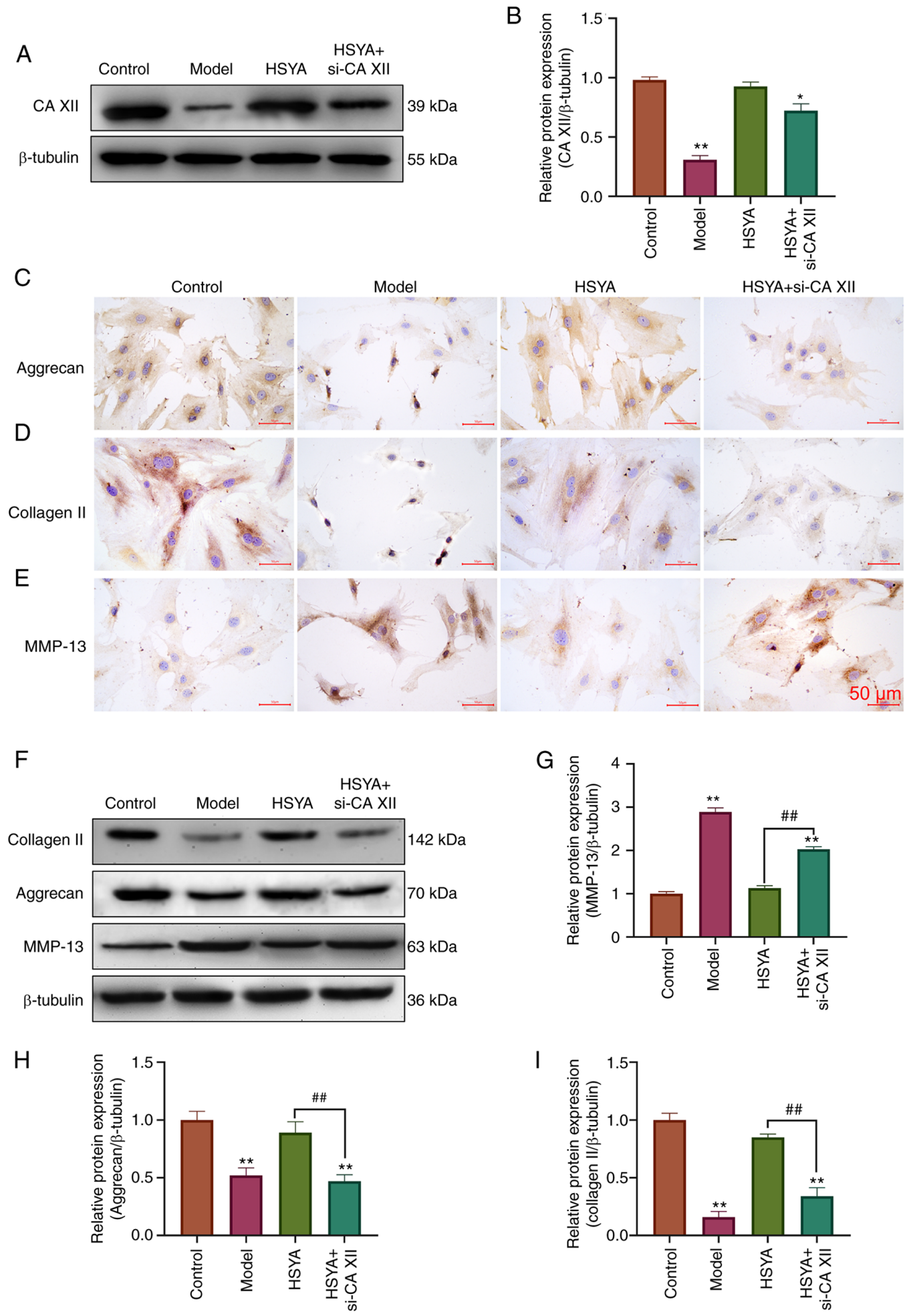

Figure 6. HSYA regulates the expression level of collagen-associated proteins in NP cells via CA XII. (A) Western blot analysis was used to detect the protein expression level of CA XII and the results were (B) statistically analyzed. Immunocytochemistry was used to detect the protein expression level of (C) aggrecan, (D) collagen II and (E) MMP-13. (F) Western blot analysis was used to detect the protein expression level of ECM-related proteins and the results were statistically analyzed for (G) MMP-13, (H) aggrecan and (I) collagen II. ${ }^{*} \mathrm{P}<0.05,{ }^{* *} \mathrm{P}<0.01$ vs. control; ${ }^{\# \#} \mathrm{P}<0.01$ vs. model. CA XII, carbonic anhydrase 12 ; HSYA, hydroxysafflor yellow A; si, small interfering.

The synthesis and degradation of the ECM maintains a dynamic balance in healthy discs, particularly in NP cells (1). However, oxidative stress disrupts this homeostasis by increasing the secretion and expression of proteases, such as MMPs and ADAMTs, and downregulating the expression of ECM proteins, such as collagen II and aggrecan (34). Therefore, the present results demonstrated that HSYA promoted the ability of NP cells to synthesize ECM components, inhibit the production of MMP-13 and reduce the catabolic level of ECM. To the best of our knowledge, we hypothesize for the first time that HSYA plays an important role in the balance between synthesis and metabolism of ECM in NP cells. 
HSYA has antioxidant and anti-apoptotic effects on NP cells, and can regulate the balance of ECM synthesis and metabolism. SEA, Pharmmappe and Moltarpred databases were used to predict HSYA target proteins. Drugbank, TTD and Genecards screened the critical proteins of disc degeneration, and those that were predicted were selected as target proteins. CA IX and CA XII were filtered from the predicted data, after which Pymol software was used to predict the binding energy of the two proteins and models, respectively. The results revealed that the highest binding energy was between HSYA and CA12. The predicted binding energy value between small molecule and protein reached $-10.7 \mathrm{kcal} / \mathrm{mol}$, which provided better conformational stability. CA XII is a transmembrane zinc metalloenzyme, from the carbonic anhydrase family that catalyze the reversible hydration of carbon dioxide into bicarbonate and hydrogen ions $(35,36)$. CA XII is considered as a marker gene of human NP cells $(20,35)$ and is enriched in human NP tissue compared with that in other tissues from the disc, including fibrosis and cartilage $(20,37)$. The study by Chen et al (18) revealed that CA XII gene and protein expression were decreased in patients with disc degeneration, while increase of CA XII expression could reduce apoptosis of NP cells and prevent degenerative disc disease. In addition, they also found that overexpression of CA XII could rescue IL-1 $\beta$-stimulated endplate chondrocyte apoptosis, both in vitro and in vitro (18). Furthermore, CA XII could also protect inner plate cartilage from the degradation of ECM and collagen regulated by the IGF-1/PI3K/CREB signaling pathway (38). CA XII inhibition may not only disrupt the $\mathrm{pH}$, which leads to lactate accumulation in NP cells, but also reduce the protein expression level of collagen II and aggrecan (20).

Therefore, RT-qPCR and western blot analysis was used to detect the gene and protein expression levels of CA XII in the NP cells. The results showed that CA XII gene and protein expression levels were significantly inhibited in the model group, while administration of HSYA could increase the gene and protein expression levels of CA XII. Therefore, the NP cells were transfected with CA XII siRNA. The results confirmed that transfection of CA XII siRNA could significantly reverse the increase of collagen II and aggrecan protein expression caused by HSYA in the NP cells, and weaken the inhibitory effect of HSYA on MMP-13 protein expression. The results were consistent with those from Chen et al (18) and Zhao et al (38). Therefore, we hypothesized that CA XII may be an important target for HSYA to exert the regulation of ECM homeostasis. However, how CA XII plays a specific role in disc degeneration requires further investigation, and there are few reports in the relevant literature. This will be the focus of subsequent research.

In summary, the present study showed that HSYA can reverse TBHP-induced NP cell apoptosis and ECM degradation. In addition, HSYA targets were predicted using bioinformatics analysis and validated using siRNA targeting CA XII. Therefore, HSYA could regulate the synthesis and metabolic balance of ECM by increasing the protein expression level of CA XII.

\section{Acknowledgements}

Not applicable.

\section{Funding}

No funding was received.

\section{Availability of data and materials}

All datasets used and/or analyzed during the current study are available upon reasonable request from the corresponding author.

\section{Authors' contributions}

SY preformed the experiments, analyzed the data and drafted the manuscript. SY and WL conceived and designed the study. WL reviewed and edited the manuscript. All authors have read and approved the final manuscript. SY and WL confirm the authenticity of all the raw data.

\section{Ethics approval and consent to participate}

The animal experiments of the current study were approved by the Ethics Committee of Affiliated Hospital of Zunyi Medical University (approval no. KLL-2020-317).

\section{Patient consent for publication}

Not applicable.

\section{Competing interests}

The authors declare that they have no competing interests.

\section{References}

1. Zhang S, Liang W, Abulizi Y, Xu T, Cao R, Xun C, Zhang J and Sheng W: Quercetin Alleviates Intervertebral disc degeneration by modulating p38 MAPK-Mediated autophagy. Biomed Res Int 2021: 6631562, 2021.

2. Trefilova VV, Shnayder NA, Petrova MM, Kaskaeva DS, Tutynina OV, Petrov KV, Popova TE, Balberova OV, Medvedev GV and Nasyrova RF: The role of polymorphisms in Collagen-encoding genes in intervertebral disc degeneration. Biomolecules 11: 1279, 2021.

3. Patil P, Niedernhofer LJ, Robbins PD, Lee J, Sowa G and Vo N: Cellular senescence in intervertebral disc aging and degeneration. Curr Mol Biol Rep 4: 180-190, 2018.

4. Wu X, Song Y, Liu W, Wang K, Gao Y, Li S, Duan Z, Shao Z, Yang $S$ and Yang C: IAPP modulates cellular autophagy, apoptosis, and extracellular matrix metabolism in human intervertebral disc cells. Cell Death Discov 3: 16107, 2017.

5. Chen S, Qin L, Wu X, Fu X, Lin S, Chen D, Xiao G, Shao Z and Cao H: Moderate fluid shear stress regulates Heme Oxygenase-1 expression to promote autophagy and ECM homeostasis in the nucleus pulposus cells. Front Cell Dev Biol 8: 127, 2020.

6. Gonzales S, Wang C, Levene H, Cheung HS and Huang CC: ATP promotes extracellular matrix biosynthesis of intervertebral disc cells. Cell Tissue Res 359: 635-642, 2015.

7. Clouet J, Fusellier M, Camus A, Le Visage C and Guicheux J: Intervertebral disc regeneration: From cell therapy to the development of novel bioinspired endogenous repair strategies. Adv Drug Deliv Rev 146: 306-324, 2019.

8. Pizzino G, Irrera N, Cucinotta M, Pallio G, Mannino F, Arcoraci V, Squadrito F, Altavilla D and Bitto A: Oxidative stress: Harms and benefits for human health. Oxid Med Cell Longev 2017: 8416763, 2017.

9. Singh A, Kukreti R, Saso L and Kukreti S: Oxidative Stress: A key modulator in neurodegenerative diseases. Molecules 24: $1583,2019$. 
10. Johnson J, Jaggers RM, Sreejit G, Dahdah A, Murphy AJ, Hanssen NMJ and Nagareddy P: Oxidative stress in neutrophils: Implications for diabetic cardiovascular complications. Antioxid Redox Signal: Jun 19, 2021 (Epub ahead of print).

11. Butterfield DA and Boyd-Kimball D: Oxidative stress, Amyloid- $\beta$ peptide, and altered key molecular pathways in the pathogenesis and progression of Alzheimer's disease. J Alzheimers Dis 62 $1345-1367,2018$

12. Masuda T, Shimazawa M and Hara H: Retinal diseases associated with oxidative stress and the effects of a free radical scavenger (Edaravone). Oxid Med Cell Longev 2017: 9208489, 2017.

13. Krupkova O, Handa J, Hlavna M, Klasen J, Ospelt C, Ferguson SJ and Wuertz-Kozak K: The natural polyphenol epigallocatechin gallate protects intervertebral disc cells from oxidative stress. Oxid Med Cell Longev 2016: 7031397, 2016.

14. Xu J, Zhan T, Zheng W, Huang YK, Chen K, Zhang XH, Ren P and Huang X: Hydroxysafflor yellow A acutely attenuates blood-brain barrier permeability, oxidative stress, inflammation and apoptosis in traumatic brain injury in rats1. Acta Cir Bras 35 e351202, 2021

15. Lee M, Zhao H, Liu X, Liu D, Chen J, Li Z, Chu S, Kou X, Liao S, Deng Y, et al: Protective effect of hydroxysafflor yellow A on nephropathy by attenuating oxidative stress and inhibiting apoptosis in induced type 2 diabetes in rat. Oxid Med Cell Longev 2020: 7805393, 2020.

16. Hu ZC, Xie ZJ, Tang Q, Li XB, Fu X, Feng ZH, Xuan JW, Ni WF and Wu AM: Hydroxysafflor yellow A (HSYA) targets the $\mathrm{NF}-\kappa \mathrm{B}$ and MAPK pathways and ameliorates the development of osteoarthritis. Food Funct 9: 4443-4456, 2018.

17. Zhang Z, Wang C, Lin J, Jin H, Wang K, Yan Y, Wang J, Wu C, Nisar M, Tian N, et al: Therapeutic potential of naringin for intervertebral disc degeneration: Involvement of autophagy against oxidative stress-induced apoptosis in nucleus pulposus cells. Am J Chin Med: Oct 4, 2018 (Epub ahead of print).

18. Chen S, Fang XQ, Wang Q, Wang SW, Hu ZJ, Zhou ZJ, Xu WB, Wang JY, Qin A and Fan SW: PHD/HIF-1 upregulates CA12 to protect against degenerative disc disease: A human sample, in vitro and ex vivo study. Lab Invest 96: 561-569, 2016.

19. van den Akker GG, Surtel DA, Cremers A, Rodrigues-Pinto R Richardson SM, Hoyland JA, van Rhijn LW, Welting TJ and Voncken JW: Novel immortal human cell lines reveal subpopulations in the nucleus pulposus. Arthritis Res Ther 16: R135, 2014.

20. Power KA, Grad S, Rutges JP, Creemers LB, van Rijen MH, O'Gaora P, Wall JG, Alini M, Pandit A and Gallagher WM: Identification of cell surface-specific markers to target human nucleus pulposus cells: Expression of carbonic anhydrase XII varies with age and degeneration. Arthritis Rheum 63 3876-3886, 2011.

21. Bayne K: Revised guide for the care and use of laboratory animals available. American Physiological Society. Physiologist 39: 199, 208-211, 1996.

22. Livak KJ and Schmittgen TD: Analysis of relative gene expression data using real-time quantitative PCR and the 2(-Delta Delta C(T)) method. Methods 25: 402-408, 2001.

23. Hu W, Zhang W, Li F, Guo F and Chen A: Bortezomib prevents the expression of MMP-13 and the degradation of collagen type 2 in human chondrocytes. Biochem Biophys Res Commun 452: 526-530, 2014

24. Lyu FJ, Cheung KM, Zheng Z, Wang H, Sakai D and Leung VY: IVD progenitor cells: A new horizon for understanding disc homeostasis and repair. Nat Rev Rheumatol 15: 102-112, 2019.
25. Swann MC, Hoes KS, Aoun SG and McDonagh DL: Postoperative complications of spine surgery. Best Pract Res Clin Anaesthesiol 30: 103-120, 2016.

26. Forrester SJ, Kikuchi DS, Hernandes MS, Xu Q and Griendling KK: Reactive oxygen species in metabolic and inflammatory signaling. Circ Res 122: 877-902, 2018.

27. Ge J, Wang Y, Yan Q, Wu C, Yu H, Yang H and Zou J: FK506 Induces the TGF- $\beta 1 /$ Smad 3 pathway independently of calcineurin inhibition to prevent intervertebral disk degeneration. Front Cell Dev Biol 8: 608308, 2020.

28. Theocharis AD, Skandalis SS, Gialeli C and Karamanos NK: Extracellular matrix structure. Adv Drug Deliv Rev 97: 4-27, 2016.

29. Guo S, Cui L, Xiao C, Wang C, Zhu B, Liu X, Li Y, Liu X, Wang D and Li S: The Mechanisms and functions of GDF-5 in intervertebral disc degeneration. Orthop Surg 13: 734-741, 2021.

30. Xu J, E XQ, Wang NX, Wang MN, Xie HX, Cao YH, Sun LH, Tian J, Chen HJ and Yan JL: BMP7 enhances the effect of BMSCs on extracellular matrix remodeling in a rabbit model of intervertebral disc degeneration. FEBS J 283: 1689-1700, 2016.

31. Gan JC, Ducheyne P, Vresilovic EJ, Swaim W and Shapiro IM: Intervertebral disc tissue engineering I: Characterization of the nucleus pulposus. Clin Orthop Relat Res 411: 305-314, 2003.

32. Le Maitre CL, Freemont AJ and Hoyland JA: Localization of degradative enzymes and their inhibitors in the degenerate human intervertebral disc. J Pathol 204: 47-54, 2004.

33. Wang J, Hu J, Chen X, Huang C, Lin J, Shao Z, Gu M, Wu Y, Tian N, Gao W, et al: BRD4 inhibition regulates MAPK, $\mathrm{NF}-\kappa \mathrm{B}$ signals, and autophagy to suppress MMP-13 expression in diabetic intervertebral disc degeneration. FASEB J 33 11555-11566, 2019.

34. Chen Y, Zheng Z, Wang J, Tang C, Khor S, Chen J, Chen X, Zhang Z, Tang Q, Wang C, et al: Berberine suppresses apoptosis and extracellular matrix (ECM) degradation in nucleus pulposus cells and ameliorates disc degeneration in a rodent model. Int J Biol Sci 14: 682-692, 2018.

35. Waheed A and Sly WS: Carbonic anhydrase XII functions in health and disease. Gene 623: 33-40, 2017.

36. Hynninen P, Hämäläinen JM, Pastorekova S, Pastorek J, Waheed A, Sly WS, Tomas E, Kirkinen P and Parkkila S: Transmembrane carbonic anhydrase isozymes IX and XII in the female mouse reproductive organs. Reprod Biol Endocrinol 2: 73, 2004.

37. Minogue BM, Richardson SM, Zeef LA, Freemont AJ and Hoyland JA: Characterization of the human nucleus pulposus cell phenotype and evaluation of novel marker gene expression to define adult stem cell differentiation. Arthritis Rheum 62: 3695-3705, 2010.

38. Zhao X, Shen P, Li H, Yang Y, Guo J, Chen S, Ma Y, Sheng J, Shen S, Liu G and Fang X: Carbonic Anhydrase 12 protects endplate cartilage from degeneration regulated by IGF-1/PI3K/CREB signaling pathway. Front Cell Dev Biol 8: $595969,2020$.

This work is licensed under a Creative Commons Attribution-NonCommercial-NoDerivatives 4.0 International (CC BY-NC-ND 4.0) License. 